\title{
Quantum-fluctuation effects on the thermopower of a single-electron transistor
}

\author{
Björn Kubala and Jürgen König \\ Institut für Theoretische Physik III, Ruhr-Universität Bochum, D-44780 Bochum, Germany
}

(Dated: May 11, 2018)

\begin{abstract}
We study thermal conductance and thermopower of a metallic single-electron transistor beyond the limit of weak tunnel coupling. Employing both a systematic second-order perturbation expansion and a nonperturbative approximation scheme, we find, in addition to sequential and cotunneling contributions, terms that are associated with the renormalization of system parameters due to quantum fluctuations. The latter can be identified by their logarithmic temperature dependence that is typical for many-channel Kondo correlations. In particular, the temperature dependence of thermopower, which provides a direct measure of the average energy of transported particles, reflects the logarithmic reduction of the Coulomb-blockade gap due to quantum fluctuations.
\end{abstract}

PACS numbers: 73.23.Hk, 73.50.Lw, 85.80.Fi

\section{INTRODUCTION}

Transport of electrons through a small metallic island is strongly affected by charging effects ${ }^{1,2,3}$ Tunneling of an electron on an island with capacity $C$ is associated with an energy of the order of the charging energy $E_{C}=e^{2} / 2 C$. At low temperature, $k_{B} T \ll \Delta$, where $\Delta$ is the charging-energy gap between ground state and first excited charge state, transport is suppressed. In a single-electron transistor (SET), an island connected to two leads by tunneling junctions (see Fig. 1), this blockade of transport can be controlled by an additional gate, resulting in the well-known Coulomb oscillations of current with respect to gate voltage. 4,5

If the island is well isolated from the leads, i.e., the barrier resistances $R_{T}^{L / R}$ are high,

$$
\alpha_{0}=\sum_{r=L, R} \alpha_{0}^{r}=\sum_{r=L, R} h /\left(4 \pi^{2} e^{2} R_{T}^{r}\right) \ll 1,
$$

electric transport is dominated by first-order transport in the tunnel conductance $\alpha_{0}$ (sequential tunneling). In the Coulomb-blockade regime, where sequential tunneling is exponentially suppressed, inelastic cotunneling becomes important. In these processes of second order in $\alpha_{0}$, the energetically unfavorable charging of the island occurs only virtually. $6,7,8,9$ But also at resonance, where sequential tunneling is present, there are higher-order transport contributions. They are associated with renormalization of charging energy and tunnel conductance due to quantum fluctuations. This can be qualitatively understood by mapping the SET at low temperature and close to resonance to a manychannel Kondo problem and performing a poor man's scaling analysis of the latter ${ }^{10}$ For a quantitative analysis of these quantum-fluctuation effects, a systematic second-order perturbation expansion within a diagrammatic real-time technique has been performed ${ }^{11,12}$ and used to study different singleelectron systems. ${ }^{13,14,15}$ In particular, a logarithmic reduction $\sim \alpha_{0} \ln \beta E_{C}$ of the maximum conductance, indicating a renormalization of the tunnel conductance, has been found, in quantitative agreement with experimental observations. 16,17

In electron transport, the transfer of charge and heat are connected to each other. This gives rise to thermoelectric ef- fects such as the thermal conductance and the thermopower

$$
S=-\left.\lim _{\delta T \rightarrow 0} \frac{V}{\delta T}\right|_{I=0}
$$

where $V$ is the voltage due to a temperature difference $\delta T$ in the absence of a charge current $I$. With the help of Onsager relations, 18 the thermopower can be related to the average energy $\langle\varepsilon\rangle$ of the transported electrons relative to the Fermi energy:

$$
S=-\frac{\langle\varepsilon\rangle}{e T} .
$$

In macroscopic conductors, the thermopower is of the order of $\left(k_{B} / e\right)\left(k_{B} T / E_{F}\right)$, which, in general, is very small. This is due to the fact that the product of density of states and electron velocity squared, determining the contribution of electrons of a certain energy to the current, varies only slowly, namely on the scale provided by the Fermi energy. Therefore, one can perform a Sommerfeld expansion of $\langle\varepsilon\rangle$. The zeroth-order term of the Sommerfeld expansion vanishes. The next-order correction, that accounts for an asymmetry of the product mentioned above around the Fermi energy, yields an extra factor of $k_{B} T / E_{F}$. This is different in mesoscopic systems for which charging effects can strongly modify the effective density of states. In the cotunneling regime of a SET, for example, the effective density of states changes on the scale

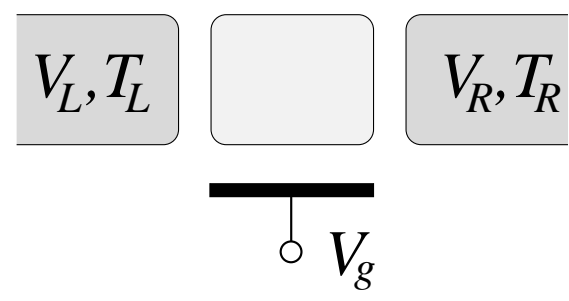

FIG. 1: Setup for thermopower measurement on a single-electron transistor. The two leads are kept at different temperatures and a voltage bias $V=V_{L}-V_{R}$ is applied, such that no electrical current flows through the device. A gate voltage sets the working point and thereby the charging-energy gap $\Delta$ of the SET. 
$\Delta$, i.e., the thermopower is of the order of $\left(k_{B} / e\right)\left(k_{B} T / \Delta\right)$. An even larger thermopower is generated by sequential tunneling processes. They are modeled by a delta-function peaked density of states around $\Delta$, for which the Sommerfeld expansion is not applicable. Instead, a direct evaluation of $\langle\varepsilon\rangle \sim \Delta$ leads to $S \sim\left(k_{B} / e\right)\left(\Delta / k_{B} T\right)$. In conclusion, at the crossover from sequential to cotunneling the thermopower can reach values of the order of $k_{B} / e$.

By now a few experiments on thermal conductance or thermopower in quantum dots have been performed 19.20.21.22.23.24.25 Thermoelectric effects in various mesoscopic systems have also been studied theoretically 26,27,28,29,30,31,32.33,34,35 Quantum dots with discrete $^{20}$ (single-particle) energy level spectrum as well as dots closer to the metallic (quasi-continuous) limit ${ }^{19}$ have been compared to sequential-tunneling theory. For increased coupling and lower temperatures, systems of discrete level structure showed signatures of cotunneling 21 and also of Kondo physics ${ }^{22}$ For the system under consideration in the present paper, a metallic single-electron transistor, thermopower has been investigated by taking into account sequential ${ }^{26}$ and cotunneling ${ }^{27}$ processes.

In this paper, we study the effect of quantum fluctuations on the thermal conductance and thermopower of a metallic single-electron transistor with a large number of transverse channels. Thermopower provides a direct access to measure a renormalization of the charging-energy gap due to quantum fluctuations since it is closely related to the average energy of tunneled electrons. As we predict below, this will give rise to a logarithmic temperature dependence of both the slope of thermopower at resonance and of the position of the maximum of thermopower as a function of gate voltage.

This work is structured as follows. First, in Sec. [II we define the model Hamiltonian of the system under consideration and derive expressions for the thermal conductance making use of two different approximation schemes. In Sec. III we discuss how the thermal conductance and the thermopower exhibit the renormalization of the charging-energy gap induced by quantum-fluctuations. We summarize our results in Sec. IV

\section{THEORY}

\section{A. The system}

A metallic single-electron transistor (see Fig. 10 is modeled by the Hamiltonian

$$
H=H_{L}+H_{R}+H_{I}+H_{\mathrm{ch}}+H_{T}=H_{0}+H_{T} .
$$

Here $H_{r}=\sum_{k n} \varepsilon_{k n}^{r} a_{r k n}^{\dagger} a_{r k n}$ and $H_{I}=\sum_{q n} \varepsilon_{q n} c_{q n}^{\dagger} c_{q n}$ describe noninteracting electrons in the two leads $r=L, R$, and on the island, respectively. The index $n=1, \ldots, N_{t}$ is the transverse channel index which includes the spin while the wave vectors $k$ and $q$ numerate the states of the electrons within one channel. In the following, we assume the many-channel limit $N_{t} \gg 1$. Coulomb interaction of the electrons on the island is described by the capacitance model $H_{\mathrm{ch}}=E_{C}\left(\hat{n}-n_{x}\right)^{2}$, where $E_{C}=e^{2} /(2 C)$ with total island capacitance $C=C_{L}+C_{R}+C_{g}$. This electrostatic energy depends on the number of excess electrons on the island, given by their number operator $\hat{n}$, as well as on applied gate and bias voltages. The latter are accounted for by the "external charge" $e n_{x}=C_{L} V_{L}+C_{R} V_{R}+$ $C_{g} V_{g}$. To increase the number of electrons in the island from $N$ to $N+1$ one has to overcome the charging-energy gap, the difference between neighboring charge states $\Delta_{N}=$ $\left\langle N+1\left|H_{\text {ch }}\right| N+1\right\rangle-\left\langle N\left|H_{\text {ch }}\right| N\right\rangle=E_{C}\left[1+2\left(N-n_{x}\right)\right]$, which is tunable by the gate voltage via $n_{x}$. The resonance condition $\Delta_{N}=0$, where the charging-energy gap vanishes, is fulfilled at half-interger values of $n_{x}$.

Finally, charge transfer processes are described by the tunneling Hamiltonian

$$
H_{T}=\sum_{r=L, R} \sum_{k q n} T_{k q}^{r n} a_{r k n}^{\dagger} c_{q n} e^{-i \hat{\varphi}}+\text { h.c. } .
$$

The matrix elements $T_{k q}^{r n} \equiv T^{r}$ are assumed to be independent of the states $k$ and $q$ and channel index $n$. They determine the tunneling resistance $R_{T, r}$ of the left and right junction by $1 / R_{T, r}=\left(2 \pi e^{2} / \hbar\right) N_{t} N_{r}(0) N_{I}(0)\left|T^{r}\right|^{2}$, where $N_{I / r}(0)$ are the density of states of the island/leads at the Fermi level. Note that, while the number $N_{t}$ of channels is large, the contribution of any one channel is so small that the total coupling remains weak and Coulomb blockade effects will occur. The operator $e^{ \pm i \hat{\varphi}}$ shifts the charge on the island by $\pm e$. Since the left and right lead are assumed to be reservoirs with fixed electrochemical potential $\mu_{r}$ and temperature $T_{r}$, the phase $\hat{\varphi}$ (or its canonical conjugate, the island charge $\hat{n}$ ) is the only independent dynamic variable in our model. In general, the electron temperatures of left lead, island, and right lead can all be different from each other and differ from the lattice temperature, $\underline{\underline{36}}$

\section{B. Conductance, thermal conductance, and thermopower}

The current $I_{r}$ flowing into reservoir $r$ can be expressed by using correlation functions for the island charge $C^{>}\left(t, t^{\prime}\right)=$ $-i\left\langle e^{-i \hat{\varphi}(t)} e^{i \hat{\varphi}\left(t^{\prime}\right)}\right\rangle$ and $C^{<}\left(t, t^{\prime}\right)=i\left\langle e^{i \hat{\varphi}\left(t^{\prime}\right)} e^{-i \hat{\varphi}(t)}\right\rangle$. For a timetranslational invariant system these correlation functions depend only on the time difference, $C\left(t, t^{\prime}\right)=C\left(t-t^{\prime}\right)$, and we will work with the Fourier transforms $C(\omega)=\int d t e^{i \omega t / \hbar} C(t)$. The tunneling current $I=I_{L}=-I_{R}$ is determined by

$$
I_{r}=-\frac{i e}{\hbar} \int d \omega\left[\alpha^{r+}(\omega) C^{>}(\omega)+\alpha^{r-}(\omega) C^{<}(\omega)\right],
$$

which includes all possible tunneling processes via the exact correlation functions $C^{\gtrless}(\omega)$. The rate functions

$$
\alpha^{r \pm}(\omega)=\alpha_{0}^{r} \int_{-\infty}^{\infty} d E f_{r}^{ \pm}(E+\omega) f^{\mp}(E)
$$

enter, $\alpha^{r+}(\omega)$ describing tunneling of an electron from lead $r$ onto the island, and $\alpha^{r-}(\omega)$ from the island to lead $r$. Here, $f^{+}$denotes the Fermi function, and $f^{-}=1-f^{+}$. Applied temperature or voltage gradients, $\delta T=T_{L}-T_{R}$ and $V=V_{L}-$ 
$V_{R}$, are accounted for by evaluating $f_{r}^{ \pm}(E+\omega)$ at temperature $T_{r}=T+\delta T_{r}$ and voltage $V_{r}$, while $f^{\mp}(E)$ is taken at $T$. We define also $\alpha(\omega)=\sum_{r} \alpha^{r+}(\omega)+\alpha^{r-}(\omega)$.

The linear electrical and thermal conductances are given by

$$
G_{V}=\left.\frac{\partial I}{\partial V}\right|_{V, \delta T=0} \quad \text { and } \quad G_{T}=\left.\frac{\partial I}{\partial \delta T}\right|_{V, \delta T=0},
$$

respectively. The thermopower describes the voltage generated by a temperature difference in the absence of an electrical current, and is related to the above mentioned conductances by

$$
S=-\left.\lim _{\delta T \rightarrow 0} \frac{V}{\delta T}\right|_{I=0}=\frac{G_{T}}{G_{V}} .
$$

To calculate the linear electrical and thermal conductance $G_{V}$ and $G_{T}$, we expand the rate functions up to linear order in either $V_{r}$ or $\delta T_{r}$ in the expression for the current, Eq. (5). It is convenient to use current conservation $\sum_{r} I_{r}=0$ to write the current as the combination $I=\left(\alpha_{0}^{R} I_{L}-\alpha_{0}^{L} I_{R}\right) /\left(\alpha_{0}^{L}+\alpha_{0}^{R}\right)$. When expanding this combination up to linear order in either $V_{r}$ or $\delta T_{r}$, we immediately see that only the equilibrium correlation functions $C^{\gtrless}(\omega)$, taken at $\delta T=0$ and $V=0$, enter, since linear corrections in $V_{r}$ or $\delta T_{r}$ drop out in the combination considered. In equilibrium, the correlation functions are related to the spectral density $A(\omega)$ for charge excitations on the island by $C^{>}(\omega)=-2 \pi i[1-f(\omega)] A(\omega)$ and $C^{<}(\omega)=2 \pi i f(\omega) A(\omega)$. This, eventually, leads to the linear electrical and thermal conductance

$$
G_{V}=G_{\text {as }} \int d \omega \frac{\beta \omega / 2}{\sinh \beta \omega} A(\omega)
$$

and

$$
G_{T}=-G_{\text {as }} \frac{k_{B}}{e} \int d \omega \frac{(\beta \omega / 2)^{2}}{\sinh \beta \omega} A(\omega),
$$

respectively, where $G_{\mathrm{as}}=1 /\left(R_{T}^{L}+R_{T}^{R}\right)$ is the classical electrical conductance asymptotically reached in the hightemperature limit. In conclusion, we need to evaluate the equilibrium spectral density $A(\omega)$ to obtain the linear electrical and thermal conductance via Eqs. (8) and (9). To keep notation simple, it is convenient to introduce dimensionless conductances $g_{V}$ and $g_{T}$, defined by

$$
g_{V}=\frac{G_{V}}{G_{\mathrm{as}}} \quad \text { and } \quad g_{T}=-\frac{e}{k_{B}} \frac{G_{T}}{G_{\mathrm{as}}} .
$$

As we see from Eqs. (8) and (9), the dimensionless conductances differ from each other by a factor $\beta \omega / 2$ in the integrand. This can be easily understood with the help of Eq. (2), which indicates that $\omega / 2$ is the average energy of the lead electrons (measured relative to the Fermi energy) that contributes to a island charge excitation of the energy $\omega$. The factor $1 / 2$ comes from averaging over the available phase space of the electronic states in the leads and the island. Roughly speaking, on average one half of charge excitation energy comes from or goes to the lead and the island electrons, respectively.

\section{Approximation schemes}

In the following we will employ two approximation schemes for calculating the spectral density and, thus, the linear electrical and thermal conductance. On the one hand, we will perform a systematic perturbation expansion up to second order in the dimensionless tunnel conductance $\alpha_{0}$. On the other hand, we will use a nonperturbative resummation scheme, the so-called "resonant-tunneling approximation" discussed further below. Both schemes go beyond the weak-coupling (sequential-tunneling) limit of small tunnel conductances, but in different ways. The virtue of either scheme as compared to the other one is discussed below. Both of these schemes are based on a real-time diagrammatic technique introduced in Ref. 37. Here, we will make use of known results of these methods without the need for an explicit recalculation of the diagrams. Therefore, in this paper, we will not discuss rules for constructing and evaluating diagrams, but refer the interested reader to the existing literature.

\section{Systematic perturbation expansion}

We perform a systematic perturbation expansion of the correlation functions $C^{\gtrless}(\omega)=\sum_{k=0}^{\infty} C^{\gtrless(k)}(\omega)$ and, therefore, automatically for the spectral density $A(\omega)=\left[C^{<}(\omega)-\right.$ $\left.C^{>}(\omega)\right] /(2 \pi i)=\sum_{k=0}^{\infty} A^{(k)}(\omega)$, where the index $k$ denotes the power of $\alpha_{0}$ in the expansion. The real-time method yields diagrammatic representations of the correlation functions in different order (see Fig. 3 of Ref.12), which are calculated as sketched in Sec. III of Ref. 12 .

To lowest order, the spectral density needed is simply

$$
A^{\mathrm{seq}}(\omega)=\sum_{N}\left(P_{N}+P_{N+1}\right) \delta\left(\omega-\Delta_{N}\right)
$$

with the equilibrium probabilities (to zeroth-order in $\alpha_{0}$ )

$$
P_{N}=\exp \left[-\beta E_{\mathrm{ch}}(N)\right] / Z \quad \text { with } \quad Z=\sum_{N} \exp \left[-\beta E_{\mathrm{ch}}(N)\right],
$$

to find the island in charge state $N$. As a result, the dimensionless linear electrical and thermal conductances are

$$
\begin{aligned}
& g_{V}^{\text {seq }}=\sum_{N}\left(P_{N}+P_{N+1}\right) \frac{\beta \Delta_{N} / 2}{\sinh \beta \Delta_{N}} \\
& g_{T}^{\text {seq }}=\sum_{N}\left(P_{N}+P_{N+1}\right) \frac{\left(\beta \Delta_{N} / 2\right)^{2}}{\sinh \beta \Delta_{N}} .
\end{aligned}
$$

For low temperatures at most two charge states contribute, e.g., for $n_{x} \approx 0.5$ only the term $N=0$ enters, and $P_{0}+P_{1}=1$. Since, in the lowest order, the only allowed charge excitation energies are $\Delta_{N}$, the average energy of the contributing electrons for transitions between charge state $N$ and $N+1$ is $\Delta_{N} / 2$.

For the next-order contribution, we use correlation functions in the limit of vanishing applied voltage and temperature bias from Ref. 12, namely Eqs. (12),(14), and (15) together with Eqs. (20) and (60) there. We can perform all integrals in Eq. (9) above analytically to find the complete second-order 
contribution to the linear electrical and thermal conductances as a sum of four terms,

$$
g_{V / T}^{(2)}=g_{V / T}^{\cot }+g_{V / T}^{\tilde{\alpha}}+g_{V / T}^{\tilde{\Delta}}+g_{V / T}^{2 e} .
$$

For the dimensionless thermal conductance, we get the lengthy but complete expressions

$$
\begin{aligned}
g_{T}^{\text {cot }}= & \sum_{N} P_{N}\left[a_{N-1} \Delta_{N-1} \partial^{2} \phi_{N-1}+a_{N} \Delta_{N} \partial^{2} \phi_{N}+\frac{a_{N}+a_{N-1}}{2} \cdot \frac{\phi_{N}-\phi_{N-1}+\Delta_{N-1} \partial \phi_{N-1}-\Delta_{N} \partial \phi_{N}}{E_{C}}\right] \\
g_{T}^{\tilde{\alpha}}= & \sum_{N} a_{N} \frac{\beta \Delta_{N} / 2}{\sinh \beta \Delta_{N}}\left(P_{N}+P_{N+1}\right)\left[\partial\left(2 \phi_{N}+\phi_{N-1}+\phi_{N+1}\right)+\frac{\phi_{N-1}-\phi_{N+1}}{E_{C}}\right. \\
& \left.+\beta \sum_{N^{\prime}} P_{N^{\prime}}\left(\phi_{N^{\prime}-1}-\phi_{N^{\prime}}\right)-\frac{\beta P_{N}\left(\phi_{N-1}-\phi_{N}\right)+\beta P_{N+1}\left(\phi_{N}-\phi_{N+1}\right)}{P_{N}+P_{N+1}}\right] \\
g_{T}^{\tilde{\Delta}}= & \sum_{N} \partial\left[a_{N} \frac{\beta \Delta_{N} / 2}{\sinh \beta \Delta_{N}}\right]\left(P_{N}+P_{N+1}\right)\left(2 \phi_{N}-\phi_{N-1}-\phi_{N+1}\right) \\
g_{T}^{2 e}= & \sum_{N} \frac{a_{N}+a_{N-1}}{2} \frac{\beta\left(\Delta_{N}+\Delta_{N-1}\right)}{\sinh \beta\left(\Delta_{N}+\Delta_{N-1}\right)}\left(P_{N-1}+P_{N+1}\right)\left[\frac{\phi_{N}-\phi_{N-1}}{2 E_{C}}-\frac{\Delta_{N-1} \partial \phi_{N}+\Delta_{N} \partial \phi_{N-1}}{\Delta_{N}+\Delta_{N-1}}\right] .
\end{aligned}
$$

Here, $\partial$ stands for $\partial / \partial \Delta_{N}=-\left(1 / 2 E_{C}\right)\left(\partial / \partial n_{x}\right)$ and we used the definition $\phi_{N}=\left(\alpha_{0}^{L}+\alpha_{0}^{R}\right) \Delta_{N} \operatorname{Re} \Psi\left(i \beta \Delta_{N} / 2 \pi\right)$, where $\Psi$ denotes the digamma function. Furthermore, we defined $a_{N} \equiv \beta \Delta_{N} / 2$. The result for the dimensionless electrical conductance $g_{V}$ is the same but without the factors $a_{N}, a_{N-1}$ and $\left(a_{N}+a_{N-1}\right) / 2$ in accordance with Ref. 12. The factors $a_{N}$ and $a_{N-1}$ account for the average energy of the lead electrons contributing to transport.

For the interpretation of the four terms we follow the reasoning put forward in Ref. 12. The first term $g_{T}^{\text {cot }}$ models cotunneling processes, where an electron is transferred through the whole device without changing the charge of the island. This is the dominant transport contribution far from the resonance of sequential tunneling. We can identify this term with the "reguralized" cotunneling result postulated in Ref. 27. In fact Eq. 14a stems from the integral expression

$$
\begin{aligned}
g_{T}^{\mathrm{cot}}=\sum_{N} P_{N} & \alpha_{0} \int d \omega \omega \frac{(\beta \omega / 2)^{2}}{2 \sinh ^{2}(\beta \omega / 2)} \\
& \times \operatorname{Re}\left(\frac{1}{\omega-\Delta_{N}+i 0^{+}}-\frac{1}{\omega-\Delta_{N-1}+i 0^{+}}\right)^{2}
\end{aligned}
$$

which yields Eq. (30) of Ref. 27 in the regime considered there. Note that the infinitesimal imaginary parts of the denominator arises naturally within the diagrammatic theory, not requiring regularization by hand as in Ref. 27. At low temperature and away from resonance, e.g., in the Coulombblockade valley with $P_{0}=1$, we can make use of the expansion $\operatorname{Re} \Psi(i x)=\ln |x|+1 /\left(12 x^{2}\right)+1 /\left(120 x^{4}\right)+\ldots$ to get

$$
\begin{aligned}
& g_{V}^{\mathrm{cot}}=\alpha_{0} \frac{2 \pi^{2}}{3}\left(k_{B} T\right)^{2}\left(\frac{1}{\Delta_{0}}-\frac{1}{\Delta_{-1}}\right)^{2} \\
& g_{T}^{\mathrm{cot}}=\alpha_{0} \frac{8 \pi^{4}}{15}\left(k_{B} T\right)^{3}\left(\frac{1}{\Delta_{0}}-\frac{1}{\Delta_{-1}}\right)^{2}\left(\frac{1}{\Delta_{0}}+\frac{1}{\Delta_{-1}}\right)
\end{aligned}
$$

in accordance with Ref. 27.

Away from resonance, $g_{V / T}^{\cot }$ is the only second-order contribution. When approaching the resonance, two more terms, $g_{V / T}^{\tilde{\alpha}}$ and $g_{V / T}^{\tilde{\Delta}}$ come into play. They are associated with sequential-tunneling processes but with renormalized parameters: $g_{V / T}^{\tilde{\alpha}}$ is the first correction term to sequential tunneling due to renormalization of the tunnel-coupling strength, $g_{V / T}^{\tilde{\Delta}}$ the respective correction due to a renormalized chargingenergy gap. The relation of these terms to renormalization is discussed in more detail below. At low temperature, only the term $N=0$ contributes and $P_{0}+P_{1}=1$, so that Eqs. 14b and (14c) reduce to

$$
\begin{aligned}
& g_{T}^{\tilde{\alpha}}=\frac{\left(\beta \Delta_{0} / 2\right)^{2}}{\sinh \beta \Delta_{0}}\left[\partial\left(2 \phi_{0}+\phi_{-1}+\phi_{1}\right)+\frac{\phi_{-1}-\phi_{1}}{E_{C}}\right] \\
& g_{T}^{\tilde{\Delta}}=\partial\left[\frac{\left(\beta \Delta_{0} / 2\right)^{2}}{\sinh \beta \Delta_{0}}\right]\left(2 \phi_{0}-\phi_{-1}-\phi_{1}\right) .
\end{aligned}
$$

The fourth term, $g_{T}^{2 e}$ describes cotunneling processes in which the charge of the island is changed by $2 e$. Since the total change of the charging energy between charge state $N+1$ and $N-1$ is $\Delta_{N}+\Delta_{N-1}$, the factor $\left(a_{N}+a_{N-1}\right) / 2$ accounts for the average energy per contributing lead electron. To overcome the charging energy for two electrons, a large temperature is required. The term $g_{T}^{2 e}$ vanishes at low temperature, and hence will not be of importance in the following.

The virtue of the perturbation expansion lies in the fact that (i) all second-order contributions are systematically taken into account, (ii) their identification with cotunneling processes and renormalization corrections to sequential tunneling is straightforward, and (iii) all expressions are unambiguously fixed by the system parameters without any remaining cutoff parameters. With increasing tunnel-coupling strength 
or lowering temperature, however, the second-order perturbation theory will become insufficient. Therefore, we also apply a different approximation scheme as described in the the following.

\section{Resonant-tunneling approximation}

The so-called resonant tunneling approximation (RTA) has been introduced in Ref. 37 as a nonperturbative treatment of quantum fluctuations. It amounts to resummation of a certain diagram class, including contributions of arbitrary high order in the tunnel-coupling strength. In particular, only two charge states $N=0,1$, and only density-matrix elements that are at most twofold off-diagonal are taken into account. For details of the derivation, we refer to Ref. 37.

Within RTA, the equilibrium spectral function is found ${ }^{37}$ to be

$$
A(\omega)=\frac{\alpha(\omega)}{\left|\omega-\Delta_{0}-\sigma(\omega)\right|^{2}}
$$

with the self-energy

$$
\sigma(\omega)=\int_{-\infty}^{\infty} d \omega^{\prime} \frac{\alpha(\omega)}{\omega-\omega^{\prime}+i 0^{+}}
$$

Real and imaginary part are given by

$$
\begin{aligned}
& \operatorname{Re} \sigma(\omega)=-2 \alpha_{0} \omega\left[\ln \left(\frac{\beta D}{2 \pi}\right)-\operatorname{Re} \Psi\left(i \frac{\beta \omega}{2 \pi}\right)\right] \\
& \operatorname{Im} \sigma(\omega)=-\pi \alpha(\omega)
\end{aligned}
$$

where $D$ is a high-energy cutoff of the order of the charging energy or band width. The expression for the electrical and thermal linear conductance follows from Eqs. (8) and (9).

The virtue of the RTA is that, due to the resummation of higher-order contributions, lower temperature and higher values of the tunnel-coupling strength are accessible. This is also indicated by the fact that the self-energy $\sigma(\omega)$, describing renormalization of the charging energy-gap and the tunnelcoupling strength, appears in the denominator of Eq. (17). On the other hand, the truncation of the Hilbert space to two charge states leaves a high-energy-cutoff dependence of the results. Thus RTA is suited for describing effects of the qualitative temperature dependence due to quantum fluctuations at low temperature. For quantitative results at higher temperature, the systematic second-order perturbation expansion is more reliable.

\section{Renormalization effects}

The main result of this paper is the appearance of renormalization effects in thermoelectric quantities. Therefore, we comment in this subsection on the relation between quantumfluctuation induced renormalization and the electrical and thermal conductance in more detail. This discussion is equally valid for the results of the electrical and thermal conductance, and follows along the line of Ref. 12 .
The notion of system-parameter renormalization is the central idea of all renormalization-group (RG) schemes. An effective low-energy model is derived by successively integrating out high-energy degrees of freedom in the leads. A poor man's scaling version of such an RG scheme for the twocharge-state approximation of the metallic SET has been performed in Ref. 10 by mapping it to a many-channel Kondo model. During the RG procedure, both the tunnel coupling strength $\alpha_{0}$ and the charging-energy gap $\Delta_{0}$ becomes renormalized as a consequence of the tunnel coupling between island and lead electrons. The renormalized values $\tilde{\alpha}$ and $\tilde{\Delta}$ are, within this poor man's scaling scheme,

$$
\frac{\tilde{\alpha}}{\alpha_{0}}=\frac{\tilde{\Delta}}{\Delta_{0}}=\frac{1}{1+2 \alpha_{0} \ln \left(D / \omega_{C}\right)}
$$

where $D$ is the high-energy cutoff (the smaller of charging energy or band width) and $\omega_{C}$ the low-energy scale at which the RG procedure stops (here the larger of temperature $k_{B} T$ or charging-energy gap $\Delta_{0}$ ). As a consequence of the large number of transverse channels in the tunnel contacts, the chargingenergy gap and the tunnel-coupling strength are renormalized towards lower values, with a logarithmic dependence on the high- and low-energy cutoff. While the result is inherently nonperturbative (an expansion of the denominator yields all powers of $\alpha_{0}$ ), it is rather qualitative as the numerical coefficient $D / \omega_{C}$ is unknown.

In the spirit of an RG picture, an effective low-energy theory of transport that takes into account renormalization is obtained by taking the sequential-tunneling formula but with renormalized system parameters $\tilde{\alpha}$ and $\tilde{\Delta}$ instead of the bare ones $\alpha_{0}$ and $\Delta_{0}$. This amounts to

$$
G\left(\alpha_{0}, \Delta_{0}\right)=G^{\mathrm{seq}}(\tilde{\alpha}, \tilde{\Delta})+\text { regular terms },
$$

for the electrical or thermal conductance. The "regular terms" represent higher-order contributions, such as cotunneling processes, that are not associated with renormalization. The latter are not included in the RG procedure, and are not considered in the following.

To relate the second-order transport contributions to renormalization, we expand Eq. (19) up to second order in $\alpha_{0}$,

$$
G^{\mathrm{seq}}(\tilde{\alpha}, \tilde{\Delta})=\frac{\tilde{\alpha}}{\alpha_{0}} G^{\mathrm{seq}}\left(\alpha_{0}, \Delta_{0}\right)+\left(\tilde{\Delta}-\Delta_{0}\right) \frac{\partial G^{\mathrm{seq}}\left(\alpha_{0}, \Delta_{0}\right)}{\partial \Delta_{0}} .
$$

By comparison with Eqs. 14b and 14cl, we obtain

$$
\frac{\tilde{\alpha}}{\alpha_{0}}=1-2 \alpha_{0}\left\{-1+\ln \left(\frac{\beta E_{C}}{\pi}\right)-\partial_{\Delta_{0}}\left[\Delta_{0} \operatorname{Re} \Psi\left(i \frac{\beta \Delta_{0}}{2 \pi}\right)\right]\right\}
$$

$$
\frac{\tilde{\Delta}}{\Delta_{0}}=1-2 \alpha_{0}\left[1+\ln \left(\frac{\beta E_{C}}{\pi}\right)-\operatorname{Re} \Psi\left(i \frac{\beta \Delta_{0}}{2 \pi}\right)\right] .
$$

Within the RTA we find ${ }^{37}$

$$
\frac{\tilde{\alpha}}{\alpha_{0}}=\frac{\tilde{\Delta}}{\Delta_{0}}=\frac{1}{1+2 \alpha_{0}\left[\ln \left(\frac{\beta D}{2 \pi}\right)-\operatorname{Re} \Psi\left(i \frac{\beta \tilde{\Delta}}{2 \pi}\right)\right]} .
$$




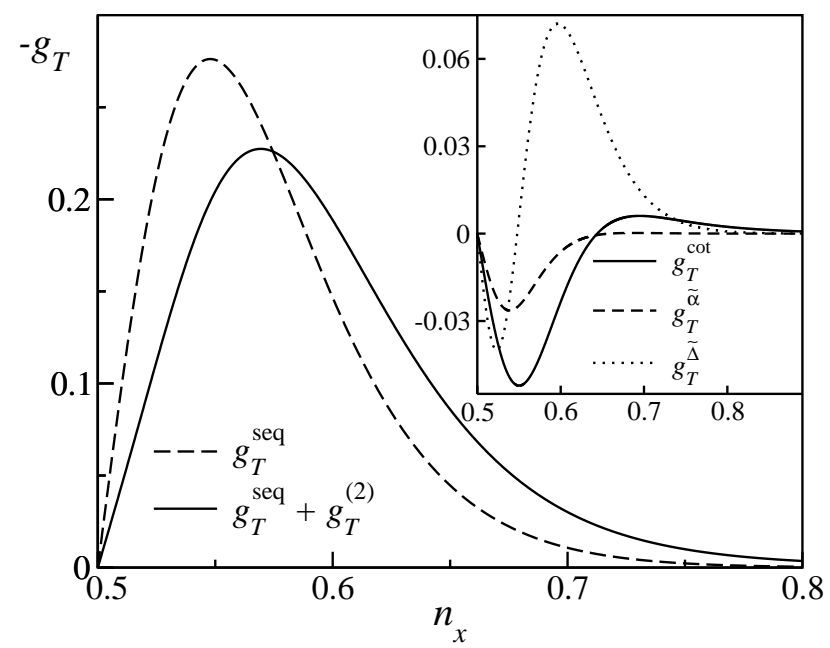

FIG. 2: Thermal conductance of an SET calculated to first and second order in tunneling coupling for $k_{B} T / E_{C}=0.05$ and $\alpha_{0}=0.04$. The inset shows the different contributions in second order, due to cotunneling $\left(g_{T}^{\text {cot }}\right)$, renormalization of the tunnel-coupling strength $\left(g_{T}^{\tilde{\alpha}}\right)$ and of the charging-energy gap $\left(g_{T}^{\tilde{\Delta}}\right)$. Renormalization leads to a broadening and a suppression of the thermal conductance as compared to the sequential-tunneling result and cotunneling yields an algebraically decaying contribution in the Coulomb-blockade valley.

The RTA result is nonperturbative in $\alpha_{0}$, and it resembles the structure of the poor man's scaling RG result in Eq. (18). Its numerical value remains undetermined as the exact form of the high-energy cutoff $D$ is left unspecified. This contrasts to the result from second-order perturbation theory. There, all numerical constants are specified. On the other hand, the renormalization is determined only up to linear corrections in $\alpha_{0}$. Indeed, this correction can be considered as the lowestorder term of an expansion in $\alpha_{0} \ln \left(E_{C} / \max \left\{\Delta_{0}, k_{B} T\right\}\right)$ [cf. Eq. [22] ], which serves as small parameter. We conclude with the remark that the interpretation of some of the second-order contributions as first-order transport but with renormalized parameters was recently supported by analyzing the full counting statistics of electrical transport through a metallic SET 38 There, the functional dependence of the cumulant generating function on the counting fields enables an unambiguous identification of sequential and cotunneling, in full support of our interpretation above.

\section{RESULTS}

\section{A. Thermal conductance}

Figure 2 shows the first- and second-order contributions to the thermal conductance, i.e., the sequential-tunneling result, Eq. (13), and the different contributions to second order, Eq. (14), in the inset. For the low temperature considered here, $g_{T}^{2 e}$ vanishes, as it corresponds to two electron processesleaving the dot in a state, where the electrostatic energy is changed by more than $E_{C}$. As discussed above, $g_{T}^{\text {cot }}$ reproduces the regularized cotunneling result; it is the dominating contribution away from resonance $\left(\beta \Delta_{0} \gg 1\right)$, as it decays algebraically only. From Eqs. (15) and (16), we see that the cotunneling contribution to the electrical and thermal conductance scales with $\left(T / \Delta_{0}\right)^{2}$ and $\left(T / \Delta_{0}\right)^{3}$, respectively. The $\left(T / \Delta_{0}\right)^{2}$ behavior of the electrical conductance is understood from the fact that each tunneling rate contributes a factor $T$ while the $\Delta_{0}^{2}$ denominator is that of standard second-order perturbation theory. At fist glance, one might expect a $T / \Delta_{0}$ behavior for the thermal conductance due to the relative factors $a_{N}=\beta \Delta_{N} / 2$ in Eq. (14a). However, it turns out that terms in the thermal conductance stemming from the lowest order in $1 /\left(\beta \Delta_{0}\right)$ in the expansion of $\phi_{0}$ cancel out when expanding Eq. 14a). Since the expansion of $\Psi(i x)$ has only even powers in $x$, the first nonvanishing contribution to the thermal conductance scales with $\left(T / \Delta_{0}\right)^{3}$.

At resonance, the terms $g_{T}^{\tilde{\alpha}}$ and $g_{T}^{\tilde{\Delta}}$, associated with renormalization of the tunnel coupling $\tilde{\alpha}$ and the charging-energy gap $\tilde{\Delta}$, become important. The renormalization of the tunnel coupling strength towards lower value results in a reduction of the peak height. The renormalization of the charging-energy gap shifts the system effectively closer to resonance and consequently yields a broadening of the resonance structure. In other words, the renormalization of coupling is reflected in the suppression of the maximum value of thermal conductance, the renormalization of the charging-energy gap in the shift of the maximum's position.

The results for thermal conductance look rather familiar from the conductance results and do not clearly showcase unexpected features. Looking at the thermopower, however, we can gain new and interesting insights in the mechanisms of electron transport through our system and how it is influenced by quantum fluctuations. This is owed to the intuitively appealing interpretation of thermopower as measure of the average energy of transported particles, see Eq. (2).

\section{B. Thermopower}

The thermopower as a function of the gate charge $n_{x}$ for different temperatures is displayed in Fig. 3 We show the full result (black lines)

$$
S=\frac{G_{T}^{\mathrm{seq}}+G_{T}^{(2)}}{G_{V}^{\mathrm{seq}}+G_{V}^{(2)}},
$$

that takes into account all first- and second-order contributions to the electrical and thermal conductance, and, for comparison, also the pure sequential-tunneling result $S^{\text {seq }}=G_{T}^{\text {seq }} / G_{V}^{\text {seq }}$ (gray lines). The thermopower vanishes at both integer and half-integer values of $n_{x}$. At resonance, i.e., at half-integer values of $n_{x}$, the thermal conductance vanishes due to a cancellation of transport contributions from lead electrons above and below the Fermi level, that generate the same charge excitation [Fig. 3]a)]. In the middle of the Coulomb-blockade valley, i.e., at integer values of $n_{x}$, the zero is due to a cancellation of processes that involve adjacent charge excitations [see Fig. 3 b)]. In between [situation sketched in Fig. 3 (c)], 

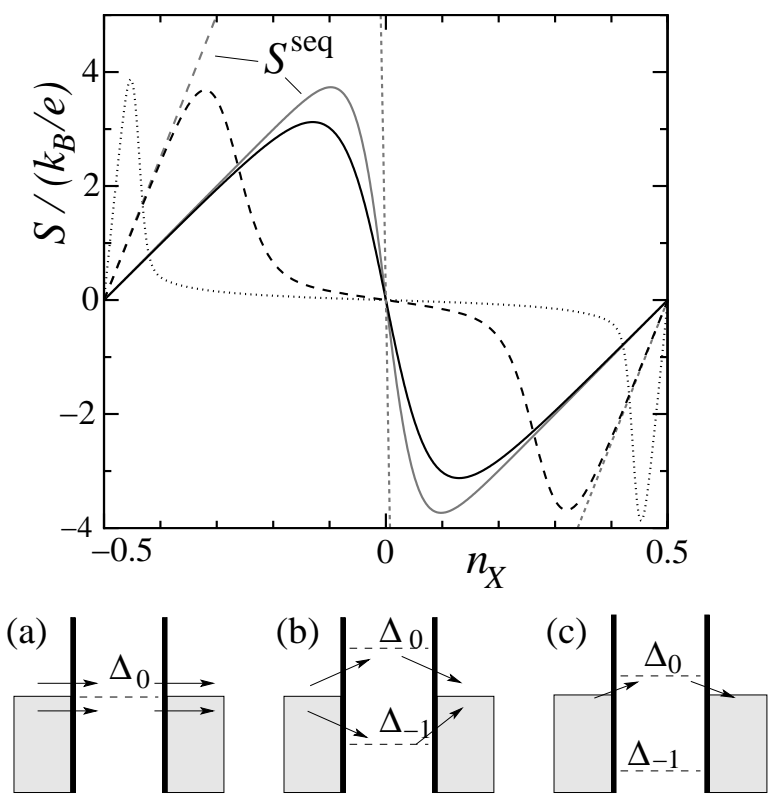

FIG. 3: Thermopower within perturbative calculation for $\alpha_{0}=0.002$ and $k_{B} T / E_{C}=0.1$ (solid line), 0.04 (dashed), and 0.01 (dotted).

the thermal conductance and, thus, the thermopower is finite, with alternating sign at integer and half-integer values of $n_{x}$.

At larger temperature (solid line in Fig. 3), sequential tunneling dominates. Results for this regime have first been derived in Ref. 26. We recover a sawtooth behavior, with a linear increase as long as transport predominantly involves only one transition $N \leftrightarrow N+1$ of charge states. Then the average energy of transported particles and correspondingly the thermopower is proportional to $\Delta_{N} / 2$. Around $n_{x} \approx N$, the adjacent transition $N-1 \leftrightarrow N$, that contributes with an opposite sign, comes into play. This gives rise to a sharply falling edge of the sawtooth with a width given by temperature.

At lower temperatures (dashed and dotted lines), sequential tunneling dominates transport only around half-integer values of $n_{x}$, but cotunneling takes over in the Coulomb-blockade valley in between, resulting in a suppression of the rising edge of the sawtooth. Instead thermopower decays with $T / \Delta$ away from resonance, as seen from Eqs. (15) and (16).

These features of the thermopower have been explained by Turek and Matveev ${ }^{27}$ by considering sequential plus cotunneling processes (the terms $g^{\text {seq }}$ and $g^{\text {cot }}$ only). They postulate a universal low- $T$ behavior, whereby $S$ scales as $S\left(\beta \Delta_{N}\right)$. We find that this does not hold true for a complete higherorder calculation. Taking into account the renormalization of system parameters due to quantum fluctuations lets the thermopower deviate from universal behavior as illustrated in Fig. [4 in which the thermopower is plotted as a function of $\beta \Delta_{0}$. Conversely, these deviations allow an insight into the renormalization process and reveal the rich physics missed by taking into account cotunneling processes only.

In the following, we will concentrate on two distinctive features of Fig. (4. On the behavior close to the resonance of se- quential tunneling (at half integer values of $n_{x}$ ) and on the position of the maximum of thermopower.

\section{Reduction of charging-energy gap}

Close to the resonance at $n_{x}=1 / 2$, transport is associated with charge excitations $0 \leftrightarrow 1$, and thermopower is linear in $n_{x}$. The sequential-tunnelig result $S^{\text {seq }}=-\left(k_{B} / e\right) \beta \Delta_{0} / 2$ corresponds to an average energy $\Delta_{0} / 2$ of the contributing lead electrons. Including cotunneling processes slightly reduces the slope (dotted line) by a factor that is independent of temperature. The slope is further reduced when taking into account all second-order contributions. Using Eq. (2) as a definition of the average energy we see that the reduction of the slope reflects a reduction of the average energy of the contributing lead electrons. In Fig. 5 we display the average energy defined via Eq. (2) close to resonance as function of temperature (this corresponds to the slope at $n_{x}=1 / 2$ in Fig. (4).

Sequential tunneling gives the ratio of $1 / 2$ for $\langle\varepsilon\rangle / \Delta_{0}$, reflecting energy averaging as discussed above (dashed line in Fig. (5). The cotunneling regularized at resonance (dotted line) yields a constant reduction by the small perturbation parameter $\alpha_{0}$ :

$$
\begin{aligned}
\frac{g_{T}^{\mathrm{seq}}+g_{T}^{\mathrm{cot}}}{g_{V}^{\mathrm{seq}}+g_{V}^{\mathrm{cot}}} & =\frac{\beta \Delta_{0}}{2} \frac{1-4 \alpha_{0}}{1-2 \alpha_{0}} \\
& =\frac{\beta \Delta_{0}}{2}\left(1-2 \alpha_{0}\right)+\mathrm{O}\left(\alpha_{0}^{2}\right) .
\end{aligned}
$$

The full next-to-leading-order calculation (solid line) of $S$ re-

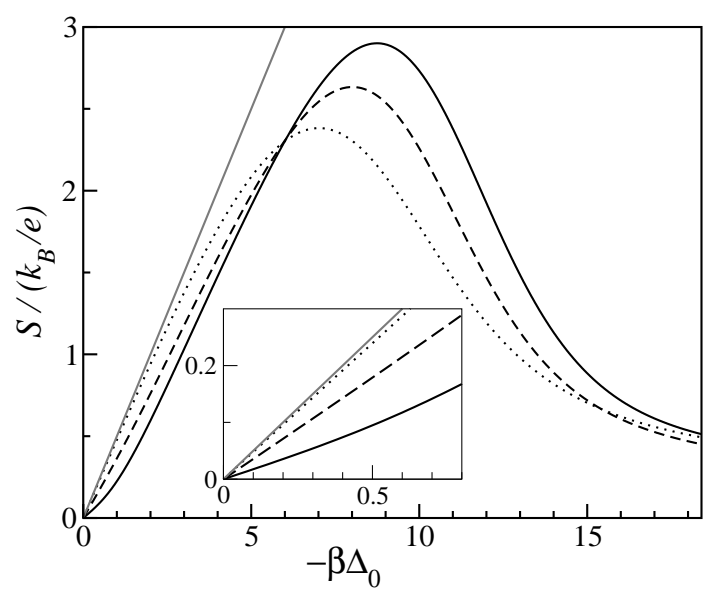

FIG. 4: Thermopower as a function of $\beta \Delta_{0}$ for $\alpha_{0}=0.02$ at low temperature. Both the curves for sequential tunneling (gray solid line) and sequential plus cotunneling (dotted line) are temperature independent. A full second-order perturbation theory, however, shows deviations from this universal scaling behavior due to chargingenergy gap renormalization. The chosen temperatures are $k_{B} T / E_{C}=$ 0.01 (dashed line) and 0.0001 (black solid line). We analyze in detail the reduction of slope at resonance (see inset) and the shift of the maximum away from resonance with decreasing temperature. 


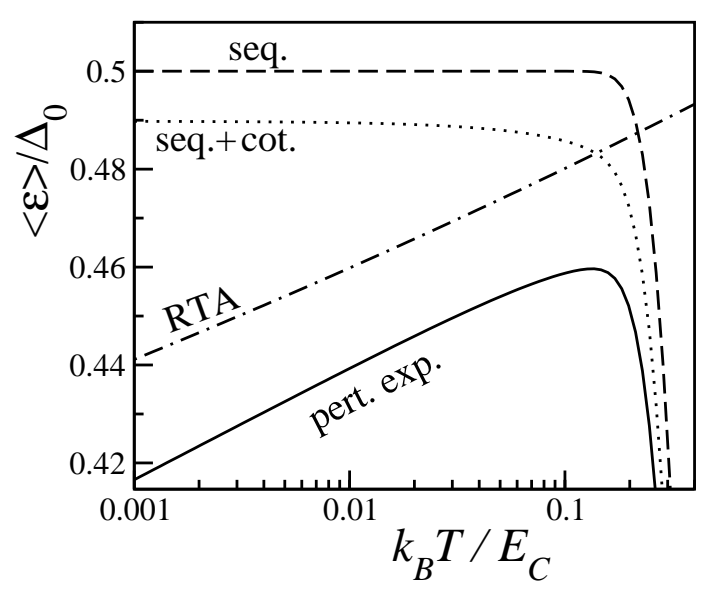

FIG. 5: Average energy of the transported particles as a function of temperature for coupling $\alpha_{0}=0.01$. The logarithmic temperature dependence typical for Kondo-physics indicates a renormalization of the charging-energy gap. We display the sequential-tunneling result (dashed line), the "universal" (temperature-independent) result of sequential and cotunneling (dotted) and the full next-to-leading-order perturbative result of Eq. (23) (solid). RTA (dot-dashed line) gives a similar low-temperature result for the slope in this Figure, but the off-set is unknown.

sults in

$$
\langle\varepsilon\rangle / \Delta_{0}=\frac{1}{2}\left[1-2 \alpha_{0}\left(2+\gamma+\ln \left(\frac{\beta E_{C}}{\pi}\right)\right)\right],
$$

with Euler's constant $\gamma=0.5772 \ldots$. The logarithmic temperature dependence directly reflects the renormalization of the charging-energy gap, cf. Eq. 21], which indicates manychannel Kondo physics.

We also show the result from the resonant-tunneling approximation (dot-dashed line), which is obtained from numerical integration of Eqs. (8) and (9) with Eq. 177. The logarithmic temperature dependence again reflects the renormalization of the level position $\tilde{\Delta}_{0} / \Delta_{0}=\left[1+2 \alpha_{0}(\gamma+\ln (\beta D / 2 \pi))\right]^{-1}$ close to resonance. While the logarithmic temperature behavior and consequently the slope in Fig. 5 is reliably predicted by RTA, the absolute vertical position depends on the choice of the high-energy cutoff (here we took $D=E_{C}$ ).

As thermopower measures the average energy of transport it yields a direct extraction of the renormalized chargingenergy gap $\tilde{\Delta}$ (via the slope of thermopower at resonance). This complements in a very appealing manner electrical conductance measurements, which reveal the renormalization of the coupling constant $\tilde{\alpha}$. As discussed in Ref. 12 and experimentally observed in Refs. 16 and 17 this renormalization of coupling is seen as logarithmic reduction of the maximal linear conductance at low temperatures.

\section{Maximum of thermopower}

The renormalization of the charging-energy gap not only modifies the slope of thermopower around $n_{x}$, it also shifts the position of maximum. Figure 6a) shows the (numerically determined) position of the maximum as a function of temperature. With only sequential and cotunneling taken into account, ${ }^{27}$ the maximum position approaches a constant when lowering the temperature (dotted line) as a consequence of the prososed unversal scaling behavior. In a full next-toleading-order theory, however, the maximum position grows logarithmically with decreasing temperature (solid line). The same low-temperature behavior is reproduced by the resonanttunneling approximation (dot-dashed line).

Since the exact analytic expression for the maximum position is not transparent, we can gain some more insight in the origin of the maximum and the temperature dependence of its position by using the following approximate treatment. Close to resonance, the average energy from sequential tunneling (and thus the thermopower) increases linearly with $\Delta_{0}$. Away from resonance, the cotunneling contribution dominates, and the thermopower decreases proportional to $T^{2} / \Delta_{0}$. The total thermopower including both types of processes is the average of the individual thermopower expression, weighted with the electrical conductances $g_{V}^{\text {seq }}$ cot

$$
-S e T=\langle\varepsilon\rangle=\frac{g_{V}^{\mathrm{seq}} \Delta_{0} / 2+g_{V}^{\mathrm{cot}}\left(k_{B} T\right)^{2} / \Delta_{0}}{g_{V}^{\mathrm{seq}}+g_{V}^{\mathrm{cot}}} .
$$

Therefore, the maximum position is roughly at the point where sequential and cotunneling electrical conductance coincide $^{27}$ (we disregard any numerical factors here).
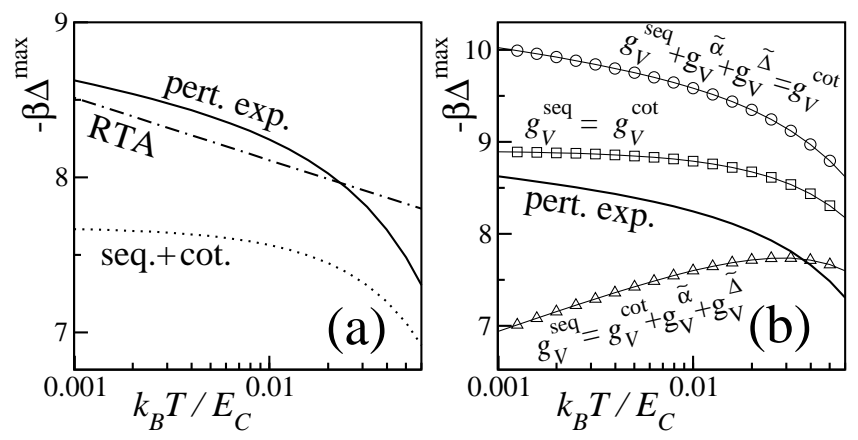

FIG. 6: (a) Position of the maximum of thermopower for $\alpha_{0}=0.01$. When taking only sequential and cotunneling into account, the maximum's position approaches a constant at low temperature (dotted line). In the full next-to-leading-order perturbative calculation (solid line) and the RTA (dot-dashed line), the maximum moves away from resonance with decreasing temperature. (b) Approximative determination of the temperature dependence of the maximum's position [thick solid line as in (a)]. The crossover between sequential and cotunneling (squares) becomes temperature independent at low-temperature. The correct temperature dependence is reproduced when the second-order terms associated with renormalization of the system parameters are added to the sequential-tunneling (circles) but not to the cotunneling (triangles) contribution. 
In Fig. @ b), we show the maximum position determined in this way. If sequential and cotunneling processes were the only contributions to be considered, the obtained maximum position is of the order of the numerically determined value and is constant at low temperature (squares). However, in a full second-order calculation there are additional terms, as discussed above. How do these terms fit into this picture? When only looking at the power of the perturbation expansion in $\alpha_{0}$ one might consider these second-order terms belonging to cotunneling. This, however, does result in a completely wrong temperature dependence (triangles). The maximum position should rather be determined by equating the cotunneling electrical conductance with that of sequential tunneling plus the extra second-order terms, that are interpreted as renormalization corrections to sequential tunneling (circles). In this case, the correct temperature dependence is reproduced. This, once again, supports the picture of renormalization.

The main effect of renormalization on the maximum position is that the peak of the electrical conductance $g_{V}^{\text {seq }}$ around resonance is broadened since the renormalized chargingenergy gap $\tilde{\Delta}=\Delta_{0}\left[1-2 \alpha_{0}\right.$ (const. $\left.\left.+\ln \beta E_{C}\right)\right]$ is reduced, i.e., the system is moved closer to resonance. As a consequence, the maximum position moves away from resonance, $\Delta^{\max }=$ $\Delta_{0}\left[1+2 \alpha_{0}\right.$ (const. $\left.\left.+\ln \beta E_{C}\right)\right]+\mathrm{O}\left(\alpha_{0}^{2}\right)$, to compensate for this renormalization, so that the renormalized maximum position is left unchanged. This is indeed the asymptotic behavior found in Fig. 6 In conclusion, the temperature dependence of the maximum position reflects the temperature-dependent renormalization of the charging-energy gap.

\section{CONCLUSIONS}

In this paper, we investigated the low-temperature properties of thermal conductance and thermopower of a metallic single-electron transistor. We presented two approximation schemes for analyzing higher-order contributions associated with quantum fluctuations: A systematic perturbative expansion in the tunnel-coupling strength and the nonperturbative resonant-tunneling approximation. Both these schemes reveal qualitatively similar physical effects and coincide for weak coupling. In particular, we find that quantum-fluctuationinduced renormalizations of the charging-energy gap and the tunnel-coupling strength affect the thermoelectric properties. They yield logarithmic temperature dependences typical for the (many-channel) Kondo effect.

For the thermal conductance, renormalization of tunnelcoupling strength and charging-energy gap results in a suppression and a broadening of the resonance features, respectively (see Fig. 2]. The effect of charging-energy-gap renormalization is most striking in the thermopower. It destroys the universal low-temperature scaling that would follow from considering sequential and cotunneling processes only (Fig. 4). The reduction of the charging-energy gap is reflected in a reduction of the slope of the thermopower at resonance, and in a shift of the maximum's position away from resonance (Figs. 5 and 6 respectively). This is due to the fact, that thermopower can be interpreted as measure of the average energy of transported particles. Therefore, an experimental observation of the charging-energy gap renormalization in the thermopower would provide an appealing complement to the tunnel-coupling renormalization measured in the electrical conductance ${ }^{16.17}$ Certainly measuring such effects in thermopower is more challenging, as precise control of temperature bias and gate voltage is required. Sequential ${ }^{19.20}$ and cotunneling ${ }^{21}$ have been observed in thermopower measurements some years after these tunneling processes had first been investigated in the electrical conductance ${ }^{1.6 .7}$ In continuing this track record also the renormalization effects on thermopower predicted in this paper may be within reach of future experiments.

\section{Acknowledgments}

We acknowledge discussions with G. Johansson and financial support by DFG via Graduiertenkolleg 726.
1 T. A. Fulton and G. J. Dolan, Phys. Rev. Lett. 59, 109 (1987).

2 O. Kulik and R.I. Shekhter, Sov. Phys. JETP 41, 308 (1975).

3 D.V. Averin and K. K. Likharev, J. Low Temp. Phys. 62, 345 (1986).

4 Single Charge Tunneling, edited by H. Grabert and M. H. Devoret, NATO Advanced Study Institute Series B: Physics (Plenum Press, New York, 1992), Vol. 294.

5 G. Schön, Single-Electron Tunneling, Chapter 3 in Quantum Transport and Dissipation, edited by T. Dittrich, P. Hänggi, G.L. Ingold, B. Kramer, G. Schön, and W. Zwerger, (Wiley-VCH Verlag, Weinheim, 1998).

6 L. J. Geerligs, D. V. Averin, and J. E. Mooij, Phys. Rev. Lett. 65, 3037 (1990).

7 T. M. Eiles, G. Zimmerli, H. D. Jensen, and J. M. Martinis, Phys. Rev. Lett. 69, 148 (1992).

8 D. V. Averin and A. A. Odintsov, Phys. Lett. A 140, 251 (1989).

9 D. V. Averin and Yu. V. Nazarov, Phys. Rev. Lett. 65, 2446 (1990).
10 K. A. Matveev, Zh. Eksp. Teor. Fiz. 99, 1598 (1991) [Sov. Phys. JETP 72, 892 (1991)].

11 J. König, H. Schoeller, and G. Schön, Phys. Rev. Lett. 78, 4482 (1997).

12 J. König, H. Schoeller, and G. Schön, Phys. Rev. B 58, 7882 (1998).

13 T. Pohjola, J. König, H. Schoeller, and G. Schön, Phys. Rev. B 59, 7579 (1999).

14 G. Johansson, A. Käck, and G. Wendin, Phys. Rev. Lett. 88, 046802 (2002).

15 B. Kubala, G. Johansson, and J. König, Phys. Rev. B 73, 165316 (2006).

16 P. Joyez, V. Bouchiat, D. Esteve, C. Urbina, and M. H. Devoret, Phys. Rev. Lett. 79, 1349 (1997).

17 C. Wallisser, B. Limbach, P. vom Stein, R. Schäfer, C. Theis, G. Göppert, and H. Grabert, Phys. Rev. B 66, 125314 (2002).

18 A. A. Abrikosov, Fundamentals of the Theory of Metals (North- 
Holland, Amsterdam, 1988).

19 A. A. M. Staring, L. W. Molenkamp, B. W. Alphenhaar, H. van Houten, O. J. A. Buyk, M. A. A. Mabesoone, C. W. J. Beenakker, and C. T. Foxon, Europhys. Lett. 22, 57 (1993).

20 A. S. Dzurak, C. G. Smith, M. Pepper, D. A. Ritchie, J. E. F. Frost, G. A. C. Jones, and D. G. Hasko, Solid State Commun. 87, 1145 (1993).

21 A. S. Dzurak, C. G. Smith, C. H. W. Barnes, M. Pepper, L. Martin-Moreno, C. T. Liang, D. A. Ritchie, and G. A. C. Jones, Phys. Rev. B 55, R10197 (1997).

22 R. Scheibner, H. Buhmann, D. Reuter, M.N. Kiselev, and L. W. Molenkamp, Phys. Rev. Lett. 95, 176602 (2005).

23 S. Möller, H. Buhmann, S. F. Godijn, and L. W. Molenkamp, Phys. Rev. Lett. 81, 5197 (1998).

24 S. F. Godijn, S. Möller, H. Buhmann, L. W. Molenkamp, and S. A. van Langen, Phys. Rev. Lett. 82, 2927 (1999).

25 X. Chen, H. Buhmann, and L. W. Molenkamp, Phys. Rev. B 61, 16801 (2000).

26 C. W. J. Beenakker and A. A. M. Staring, Phys. Rev. B 46, 9667 (1992).

27 M. Turek and K. A. Matveev, Phys. Rev. B 65, 115332 (2002).

28 A. V. Andreev and K. A. Matveev, Phys. Rev. Lett. 86, 280
(2001).

29 K. A. Matveev and A. V. Andreev, Phys. Rev. B 66, 045301 (2002).

30 J. Koch, F. von Oppen, Y. Oreg, and E. Sela, Phys. Rev. B 70, 195107 (2004).

31 D. Boese and R. Fazio, Europhys. Lett. 56, 576 (2001).

32 J. P. Small, K. M. Perez, and P. Kim, Phys. Rev. Lett. 91, 256801 (2003).

33 M. Turek, J. Siewert, and K. Richter, Phys. Rev. B 71, 220503(R) (2005).

34 Ya. M. Blanter, C. Bruder, R. Fazio, and H. Schoeller, Phys. Rev. B 55, 4069 (1997).

35 G. D. Guttman, E. Ben-Jacob, and D. J. Bergman, Phys. Rev. B 51, 17758 (1995).

36 R. L. Kautz, G. Zimmerli, and J. M. Martinis, J. Appl. Phys. 73, 2386 (1993).

37 H. Schoeller and G. Schön, Phys. Rev. B 50, 18436 (1994); J. König, H. Schoeller, and G. Schön, Europhys. Lett. 31, 31 (1995).

38 A. Braggio, J. König, and R. Fazio, Phys. Rev. Lett. 96, 026805 (2006). 